\title{
BMJ Open Randomised controlled trial of a video intervention and behaviour contract to improve medication adherence after renal transplantation: the VECTOR study protocol
}

\author{
Holly Mansell,, ${ }^{\oplus}$ Nicola Rosaasen, ${ }^{2}$ Patricia West-Thielke, ${ }^{3}$ Jenny Wichart, ${ }^{4}$ \\ Christopher Daley, ${ }^{5}$ Rahul Mainra, ${ }^{6}$ Ahmed Shoker, ${ }^{6}$ Juxin Liu, ${ }^{7}$ David Blackburn ${ }^{1}$
}

To cite: Mansell H, Rosaasen N, West-Thielke P, et al. Randomised controlled trial of a video intervention and behaviour contract to improve medication adherence after renal transplantation: the VECTOR study protocol. BMJ Open 2019;9:e025495. doi:10.1136/ bmjopen-2018-025495

- Prepublication history for this paper is available online. To view these files, please visit the journal online (http://dx.doi. org/10.1136/bmjopen-2018025495).

Received 17 July 2018 Revised 5 December 2018 Accepted 25 January 2019

Check for updates

(C) Author(s) (or their employer(s)) 2019. Re-use permitted under CC BY-NC. No commercial re-use. See rights and permissions. Published by BMJ.

For numbered affiliations see end of article.

Correspondence to Dr Holly Mansell; holly.mansell@usask.ca

\section{ABSTRACT}

Introduction Non-adherence after kidney transplantation contributes to increased rejections, hospitalisations and healthcare expenditures. Although effective adherence interventions are sorely needed, increasing education and support to transplant recipients demands greater use of care providers' time and resources in a healthcare system that is stretched. The objective of this clinical trial is to determine the effectiveness of an electronically delivered video series and adherence behaviour contract on improving medication adherence to immunosuppressant medications.

Methods and analysis A multicentre, parallel arm, randomised controlled trial will be conducted with four sites across North America (Saskatoon, Calgary, Halifax, Chicago). Adult patients will be randomised (1:1) to either the intervention (ie, home-based video education +behaviour contract plus usual care) or usual care alone. De novo transplant recipients will be enrolled prior to their hospital discharge and will be provided with electronic access to the video intervention (immediately) and adherence contract (1 month post-transplant). Follow-up electronic surveys will be provided at 3 and 12 months postenrolment. The primary outcome will be adherence at 12 months post-transplant, as measured by self-report Basel Assessment of Adherence to Immunosuppressive medications and immunosuppressant levels. Secondary outcomes include the difference in knowledge score between the intervention and control in groups (measured by the Kidney Transplant Understanding Tool); differences in self-efficacy (Generalised Self-efficacy Scale), Beliefs of Medicine Questionnaire (BMQ), quality of life (Short Form-12), patient satisfaction and cost utilisation. The study aims to recruit at least 200 participants across participating sites.

Ethics and dissemination Ethical approval was obtained from the University of Saskatchewan Behavioural Ethics Committee (Beh 18-63), and all patients provide informed consent prior to participating. This educational intervention aims to improve information retention and self-efficacy, leading to improved medication adherence after kidney transplantation, at low cost, with little impact to existing healthcare personnel. If proven beneficial, delivery can be easily implemented into standard of care.

\section{Strengths and limitations of this study}

- This multicentre study employs a randomised, controlled design.

- The intervention, which does not require substantial resources to deliver, incorporates an educational component (which was developed with patients) and a behavioural strategy to support autonomy, an important aspect of medication adherence.

- The main limitation is the lack of power to evaluate clinical outcomes such as graft function or mortality; however, the primary endpoint is adherence to medications, which has been directly linked to major clinical events.

- Subjects in the experimental group cannot be blinded due to the nature of the educational interventions, and may be prone to a Hawthorne effect.

Trial registration number NCT03540121; Pre-results.

\section{INTRODUCTION}

Kidney transplantation is the gold-standard treatment for patients with end-stage renal disease, and the benefits are immense compared with dialysis. Transplantation is associated with a better quality of life and patients live two to three times longer. ${ }^{1-3}$ However, following the transplant, patients must commit to indefinite therapy with immunosuppressive medications. Failure to adhere to these medications can result serious consequences, such as graft rejection, graft loss and significant costs to the healthcare system. ${ }^{4}$ Approximately one out of five transplant recipients fail to take their regimen as prescribed. ${ }^{5}$ Consequently, up to one-third of all solid organ rejections may be precipitated by non-adherence. ${ }^{6}$ Considering that more than 30000 kidney transplants are 
performed yearly in the USA alone, ${ }^{7}$ medication non-adherence is a problem that must be taken seriously.

Immunosuppressive therapy is initiated at the time of transplant surgery, leaving little time for patients to prepare for the reality of multiple medication use following hospital discharge. The early postoperative period is often associated with anxiety and confusion, and may not be the most effective time for patients to receive information. ${ }^{8-11}$ However, due to logistical factors, medication and lifestyle teaching is often performed by the healthcare team prior to the patient's discharge. Even though patients are educated during the transplant process, poor knowledge about medications remains a major problem following transplant surgery ${ }^{11-13}$ for reasons largely unknown. Furthermore, patient self-efficacy for taking medications and medication adherence wanes over time. ${ }^{14}$

Effective educational opportunities can help patients prepare for the complexities of transplantation and may positively influence outcomes. The evidence, however, is strongest in the pretransplant period, where a number of well-designed trials have recently been undertaken. Literature has illustrated the benefits of using educational strategies to improve knowledge and willingness to discuss living organ donation, as well as predicting success at identifying donors. ${ }^{15-18}$ Video and printed education has also been effective at increasing willingness to serve as an organ donor in ethnic populations where donation rates are typically low. ${ }^{19}$ Nevertheless, well-designed interventional studies examining educational outcomes after transplant are rare. Randomised controlled trials (RCTs) investigating adherence as an outcome have historically been even more uncommon.

Successful adherence interventions have generally employed a multidimensional strategy including education and counselling on medication taking, discussion of adherence barriers and goal setting. ${ }^{20}$ Adherence interventions often include strategies to promote self-efficacy and have linked to improved confidence, empowerment, ${ }^{8}$ medication adherence and quality of life. ${ }^{21} 22$ Chisholm-Burns and colleagues explored the impact of using education from a clinical pharmacist in combination with a behavioural contract (patient agreement to work on adherence), compared with usual care. ${ }^{23}$ Significantly higher adherence rates were noted in the intervention group, along with decreased hospitalisations at 12 months. However, the intervention involved individualised collaboration between patients and a study pharmacist, necessitating additional resources and time. Hence, replication into other settings may be challenging.

'Solid Organ Transplantation: An Educational Mini-Series for Patients' is a patient-centred video series that describes the kidney transplant process. It was developed with patients, for patients and input from additional patient stakeholders was provided throughout the development process. Best practice for designing education for patients pursuing transplant was used ${ }^{24}$ and a multidisciplinary team including experts in medication adherence, video education, motivational psychology, cultural education and healthcare providers were involved in this process. A manuscript detailing the video development process has been published elsewhere. ${ }^{25}$ The videos consist of animated segments which accommodate patients with low health literacy. Narratives by actual patients and healthcare providers help personalise the information, and provide real world advice, aligning the content with principles of the adult learning theory, which states that adults should be involved in the planning and evaluation of the learning, and are most interested in topics that have personal relevance and are problem centred. ${ }^{26}{ }^{27}$ Video-based education has been shown to be at least as effective as traditional methods of education, ${ }^{28} 29$ and allows the opportunity for information to be consistently delivered, and replayed as often as needed. ${ }^{30-32}$

We hypothesise that patient-centred video education combined with an adherence contract, will enhance information retention about medications, and self-efficacy, leading to improved medication adherence to immunosuppressive medications after kidney transplantation. The intervention will be provided electronically to supplement routine interactions with the transplant program, without additional heavy burden on health providers who are already have a high workload. Furthermore, patients will have the opportunity to watch and replay information as desired in the comfort of their home.

\section{METHODS AND ANALYSIS}

The Video Education and Behaviour Contract to Optimize Adherence in Renal Transplants (VECTOR) study is a multicentre, parallel design RCT that will be conducted to test the effectiveness of the video intervention plus adherence contract on improving kidney transplant candidate's medication adherence to immunosuppressive medications, compared with usual care. Four transplant sites across North America will be participating in this study: the Saskatchewan Transplant Program (located in Saskatoon, SK), Southern Alberta Transplant Program (Calgary, AB), Multi-organ Transplant Program of Atlantic Canada (Halifax, NS), University of Illinois Health Sciences System (Chicago, IL). A study lead at each site will take responsibility for local research ethics submission, recruitment, enrolment and follow-up activities.

\section{Patient recruitment, eligibility and enrolment}

Patients who have received a de novo kidney transplant at one of the study sites during the enrolment period will be invited to participate in the study. Eligible patients will be 18 years or older, and can speak, understand and read English (or have a support person that helps them). Participants will be excluded if they have previously received a kidney transplant, or have a visual along with a hearing impairment that would absolutely prohibit viewing the educational materials. Many transplant recipients 
experience language barriers or other impairments that can impede the delivery of education. These individuals usually rely on a designated support person to assist with the transplant process and their postoperative care. In order to represent the population cohort and 'real life' as accurately as possible, we will accept participation from individuals who need assistance with completing the tasks, provided that a designated support person is able to assist them, and an adequate accommodation can be made. (For instance, in the case of a language barrier, participation may be feasible with the assistance of a family member. Or, in the case of a hearing impairment, closed captioning can be turned on for participants randomised to the intervention group.) Finally, patients who have previously participated in another study entitled: 'Improving health outcomes of kidney recipients: A Randomized controlled trial of a pre-transplant education intervention', which is taking place at two of the study sites $(\mathrm{SK}, \mathrm{AB})$ will also be excluded, since this study uses a version of the video intervention in a pretransplant cohort. The importance of keeping all study materials confidential is stressed to patients participating in both studies, to minimise the possibility of contamination.

During the transplant hospitalisation (as the patient is getting ready for discharge), a healthcare provider will explain the purpose of the study and assist patients in enrolling by setting up a study account using a study iPad. Depending on the site's workflow, the 'enroller' may be a healthcare provider (eg, nurse, pharmacist, nurse or physician), or study coordinator. A standardised script will be used to ensure consistency between all enrollers and sites. The study lead at each site will follow a strict protocol for maintaining patient data to prevent any breach of privacy/confidentiality.

\section{Randomisation and blinding}

Following consent, patients will be randomised (1:1) to either the intervention (ie, home-based video education plus a behaviour contract plus usual care) or usual care alone, using pregenerated survey participation links. Randomisation will be performed in permuted blocks of either six or eight, stratified according to site a custom Python (V.2.7.11) script. A third-party research organisation, the Social Science Research Laboratory (SSRL) at the University of Saskatchewan, will perform the randomisation centrally and provide sequentially numbered survey participant links to the study sites, that will conceal the treatment.

The study team will remain blind to participant allocation. Participant blinding is not possible in behavioural studies, however, every attempt will be made to treat the two groups consistently to minimise bias. Participants in both groups will be informed that they will be randomised to one of two groups, and that the intervention group will receive 'extra education and materials about taking medication'. They will not be explicitly told what the intervention entails. Further, patients in the control group will receive communications of similar content and frequency to the intervention group to help maintain consistency. Patients in the trial will be reminded not to discuss the nature of the intervention with friends or the program staff, or other patients. They will also be provided with a personalised, password-protected unique login credentials to access the video content and prevent uncontrolled sharing of the video links.

\section{Baseline questionnaire}

Participants that provide consent will be asked to complete a baseline questionnaire on the study iPad. The questionnaire assesses demographic and clinical information such as age, sex, race/ethnicity, levels of education, whether or not a patient is on dialysis and what type (ie, haemodialysis or peritoneal), marital status, resident location, travel time to transplant centre, self-reported adherence and health literacy. Similar to previous research in transplantation, health literacy will be assessed with two items scored on Likert-type scales: 'How often do you have someone (like a family member, friend, hospital/clinic worker or caregiver) help you read hospital materials?', and 'How confident are you filling out forms by yourself?'. ${ }^{334}$ Baseline transplant knowledge will be measured by the Kidney Transplant Understanding Tool (K-TUT), while self-efficacy, beliefs of medicine and quality of life will be measured by the Generalised Self-efficacy Scale (GSE), Beliefs About Medicines Questionnaire (BMQ) and Short Form-12 (SF-12) scales, respectively. ${ }^{35-38}$

\section{Intervention: standard of care + video education + behaviour contract}

Participants randomised to the intervention group will receive standard education along with the home-based video education plus an adherence contract. Following the baseline assessment, access to videos will be provided. A personalised, password-protected link is provided to the participant, and the videos are accessible using this link from any electronic device. Participants may view them on a device in the hospital and/or they can watch and replay them at home. Patients who wish to view the videos in the hospital setting are provided with headphones to protect the participant's privacy and study contamination. The link is active throughout the duration of the study so that the videos can be accessed as often as desired. The videos are part of a series entitled 'Solid Organ Transplantation: An Educational Mini-Series for Patients', which consists of six videos outlining all stages of the transplant process. In the present study we will be using a modified version of the series consisting of three of the videos; Video 1: Introduction, Video 2: Medications, Video 3: Your New Life. The first video sets the stage for the intervention and introduces the viewer to the series. An animated overview of the immune system is presented and some commonly used transplant terms are discussed. The second video provides an overview of commonly used transplant immunosuppressants. It discusses common side effects and provides information on how to cope with lifelong medication taking. Testimonials from actual patients and 


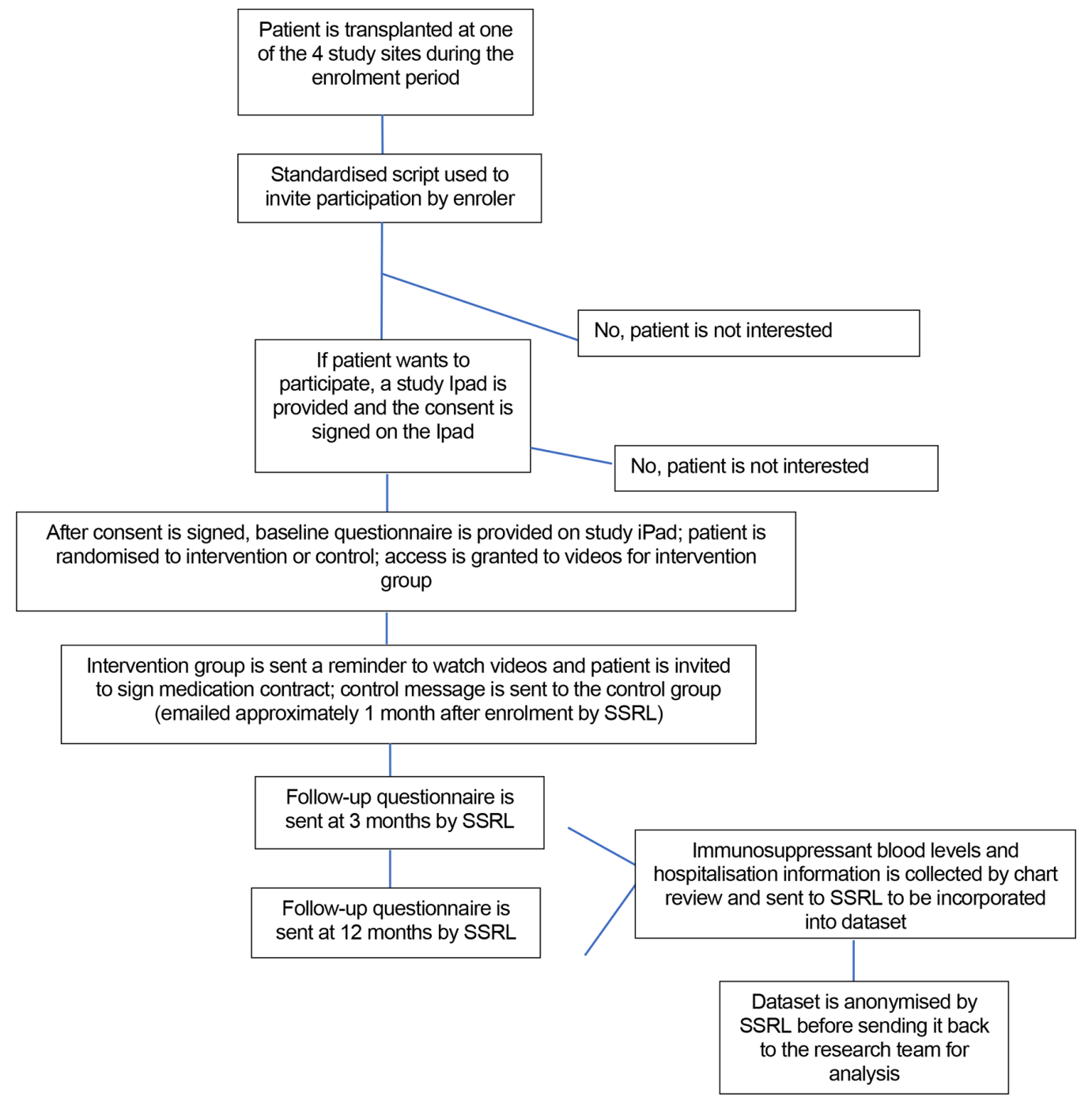

Figure 1 Depicts a flow chart describing the study process. SSRL, Social Sciences Research Laboratory.

a donor family supplement the information and provide patient perspectives on medication adherence. The final video addresses common questions that patients have about life after transplant, which were curated from actual patients. Some of the topics pertain directly to post-transplant health (such as infection prevention, cancer screening and risk minimisation, post-transplant monitoring and medication-related questions), while others are more general in nature (such as exercise, returning to work, how to honour your donor). Three of the videos from the original series will not be shown because they pertain to issues pretransplant. The SSRL will be monitoring video viewing statistics throughout the course of the study. Patients in the intervention group who have not accessed the videos prior to the 1-month behavioural contract will receive reminders to do so.

Approximately 1 month after the patient has been enrolled into the study and been provided with access to the videos, participants will receive an email link inviting them to reflect on their goals about transplantation and pledging to taking their immunosuppressant medications as directed. The adherence contract used in this study was inspired by previous research performed by ChisholmBurn and colleagues. ${ }^{23} 39$ The participant is asked to consider the health goal of remaining adherent to his or her immunosuppressive therapy regimen, and reflect on motivation, and consequences of failing to perform the behaviour, and then set an action plan for taking medications as directed. Example goals are provided to the participant, along with free-formed text boxes so that the participant can type in their own personal responses. Although the goal setting aspect of the intervention is somewhat visionary in nature (ex: 'My health goal is to be adherent to my immunosuppressant therapy regimen to remain healthy for my family'), the participant is asked to provide a specific plan to achieve the goal, and to consider problems that may interfere with reaching the goal, along with potential solutions. The contract will be non-enforceable (no penalties or rewards are provided for contract adherence), but it will provide patients with the opportunity to formally commit to a goal of taking the medications as prescribed. A signature box has been programmed into the software, so that participants can provide a personal handwritten signature. The behaviour 
Table 1 Summary of interventions, assessments and data collected at specified time points

\begin{tabular}{|c|c|c|c|c|}
\hline & $\begin{array}{l}\text { De Novo Transplant } \\
\text { Admission }\end{array}$ & $\begin{array}{l}1 \text { month after } \\
\text { transplant }\end{array}$ & $\begin{array}{l}3 \text { months after } \\
\text { transplant }\end{array}$ & $\begin{array}{l}12 \text { months after } \\
\text { transplant }\end{array}$ \\
\hline $\begin{array}{l}\text { Demographic and medical } \\
\text { characteristics }\end{array}$ & $\sqrt{1}_{1}$ & & & \\
\hline $\begin{array}{l}\text { Prior education regarding transplant } \\
\text { and health literacy }\end{array}$ & $V_{1}$ & & & \\
\hline Knowledge assessment & $V_{1}$ & & $V_{1}$ & $\sqrt{1}_{1}$ \\
\hline Video viewing habits & & $\sqrt{3}$ & $\sqrt{3}$ & $\sqrt{3}$ \\
\hline Satisfaction with education & & & $V_{1}$ & $V_{1}$ \\
\hline Self-efficacy & & & $V_{1}$ & $V_{1}$ \\
\hline Beliefs of medicine & & & $\sqrt{1}_{1}$ & $\sqrt{1}_{1}$ \\
\hline Days in hospital & & & & $\sqrt{2}_{2}$ \\
\hline
\end{tabular}

$V_{1}=$ self-report questionnaire sent by SSRL; $\sqrt{2}_{2}=$ data collected and submitted by study site; $V_{3}=$ data populated by study software.

intervention uses self-reflection and the contract is completed autonomously, without health care provider witness or interference. The participant is encouraged to contact their transplant team if they require any support with taking their medications. However, since the intent is to test an intervention that can be delivered without substantial resources, and minimise bias that goal coaching may introduce in this multicentre trial, the contract is not formally reviewed with the transplant centre. The goal setting activity is revisited with participants during the 3 months and 12 months postenrolment surveys.

Based on Saskatchewan data, ${ }^{40}$ over $90 \%$ of transplant candidates have access to the internet and will be able to obtain the intervention and outcome assessments in the form of surveys over a computer from home. Videos will be distributed electronically to all intervention participants by the SSRL using a password protected link. The SSRL has extensive experience in overseeing experimental research activities and will manage the study for the duration of the follow-up period, allowing a standardised process that will be used across all four sites. They will disseminate videos, administer surveys and collect data and deidentify the final dataset prior to analysis by the research team. Participants that do not have access to the internet at home will be able to view the videos electronically, access the questionnaires and sign the contract when they present to the transplant clinic for their routine post-transplant appointments, using their phone and the hospital's Wi-Fi, or the study iPad. The SSRL will streamline this process by providing a site-specific list of patients requiring face to face follow-up during appointments. Study site leads will ensure that participants are followed up at key check points (1 month, 3 months and 12 months postenrolment).

\section{Control: standard of care education}

The control group will receive the standard of care education that is routinely provided at each transplant centre. In all centres, this includes verbal and written information provided by the healthcare team. Before discharge all patients receive 1:1 medication education with a healthcare provider (nurse or pharmacist) as per a standard teaching checklist. Anything not covered before discharge will be flagged as an outstanding teaching point to the ambulatory transplant pharmacist. Medication teaching sheets and a personalised medication schedule are also provided. Patients randomised to the control arm will receive the same assessments throughout the study period, without access to the videos. In lieu of the adherence contract, participants in the control group will receive an email message thanking them for participating in the study, and explaining that the next assessments (surveys) will occur at 3 months and 12 months after enrolment.

\section{OUTCOME MEASURES}

\section{Primary outcome: medication adherence}

The primary outcome will be the difference in the percentage of participants adherent to their immunosuppressive medications between the intervention and control groups at 1-year post-transplant. Adherence to immunosuppressant medications will be measured by using self-report using the Basel Assessment of Adherence to Immunosuppressive medications sent electronically to each participant. ${ }^{41}$ An answer of 'yes' to any of 
the questions (pertaining to missing doses, drug holidays, timing and dose reduction) will constitute non-adherence as a binary outcome.

Two additional measures will also be assessed, a visual analogue scale (VAS) and immunosuppressant blood levels. The VAS will provide a continuous measure of adherence collected through the electronic survey while outpatient immunosuppressant levels (tacrolimus, cyclosporine or sirolimus) will be collected as per routine practice, and will be standardised to the patient-specific target. Intrapatient coefficients of variation will be used as a measure of trough level variability, and percentage of subtherapeutic levels will be determined for each patient. The frequency of immunosuppressant monitoring varies depending on the time post transplant, with more intense monitoring performed early in the transplant period (ex. Two or three times per week for up three months post-transplant, and around once monthly closer to 1-year post-transplant.). In all centres, immunosuppressant monitoring is performed at least monthly during the first year post-transplant. This information will be calculated and reported to SSRL by site leads. All measures described above have been validated in transplant literature and have been significantly associated with rejection. $^{42} 43$

\section{Secondary outcomes}

Secondary outcomes include the difference in change in knowledge score between the intervention and control groups, and differences between the intervention and control in the following measures: self-efficacy, beliefs about medications, quality of life, satisfaction with education, adherence to appointments and days in hospital. Knowledge of kidney transplant will be measured by the K-TUT, which has been evaluated in both pretransplant and post-transplant cohorts, and has been shown to have good internal consistency, reproducibility, content and construct validity. ${ }^{35}$ Self-efficacy will be captured using the GSE which assesses beliefs for coping with difficult demands in life. ${ }^{36}$ It has been used in many disease states, including transplantation. ${ }^{44}{ }^{45}$ Beliefs about medications will be measured using the BMQ, which measures cognitive perceptions of a patient's own medications and beliefs in general. ${ }^{37}$ It has been validated in patients with chronic illnesses. ${ }^{46}$ Quality of life will be measured by the SF-12 V.2 (SF-12®) ${ }^{38}$ a validated short version of the SF-36. The SF-12 has been used in several kidney transplant cohorts. ${ }^{47-49}$ Satisfaction about the educational experience will be captured by five questions whereby participants rate their confidence, understanding and satisfaction with education about transplant medications and lifestyle on a Likert scale of 1-5. The above described measures will be collected by way of a self-reported survey administered 3 months and 12 months post-transplant, either at home electronically or on the clinic iPad. The data will be immediately transmitted to SSRL.

Adherence to appointments or 'no shows' are defined as the percentage of times a patient failed to present to an appointment, or scheduled test without calling ahead. In addition, postintervention hospitalisation rates will be collected to estimate potential reductions in usage and costs associated with the intervention. Further, costs of the intervention will be compared with gains in utility based on the SF12. Adherence to appointments, staff time in administering the intervention and days in hospital during the study period will be collected retrospectively at the study end and reported to the SSRL by the study site leads. ${ }^{50}$ The site leads will remain blind to participant allocation and the SSRL will incorporate the information into the final dataset and deidentify it before providing it to the research team for analysis.

In the intervention group, individualised viewing statistics will be available from the SSRL allowing an assessment of video viewing habits. The total duration that participants have watched each video in minutes will be recorded and will be divided by the total length of each video to be used as a surrogate marker for whether the video was watched in its entirety. The percentage of patients viewing at least one module, percentage of patients viewing the mini-series in its entirety will be reported. Three additional questions will be used to collect information on whether participants liked or disliked the intervention, and what aspects they believe should be changed. Figure 1 depicts a flow chart of the study process, table 1 describes a summary of the interventions, assessments and data collected at specific time points during the study process.

\section{Incentives}

Study participants will be provided with incentives in the form of gift cards during specific time points in the study to encourage continued participation. A \$20 gift card will be provided after the study participants complete the 3-month post-transplant questionnaire. A $\$ 25$ gift card will be issued for participants who reach the study endpoint and complete the final survey. Although no penalties are issued for participants in the intervention group that fail watch the videos or complete the behavioural contract, their participation is monitored.

\section{Sample size}

The primary endpoint is the percentage of patients reporting optimal adherence to their immunosuppressants at one year post-transplant. A sample of 100 patients in each group is sufficient to determine a $15 \%$ absolute increase (from $75 \%$ to $90 \%$ ) in percentage of adherent patients at an alpha of 0.05 and a beta of 0.20 . This sample will allow for exploration of smaller changes $(10 \%)$ in secondary analyses of mean adherence measured by the visual analogue scale. Thus, assuming a drop-out rate of up to $25 \%$, we will enrol 268 patients to ensure the primary analysis is adequately powered.

\section{Data analysis}

All patients who have completed at least one postbaseline assessment will be included in the primary endpoint 
analysis. The percentage of patients in each group reporting optimal adherence will be compared using a $\chi^{2}$ test. In cases where the final assessment is missing, the last observations are carried forward. In a secondary analysis, the additional adherence measures will be analysed and triangulated with the primary endpoint comparison. Also, the primary endpoint comparison will be repeated including all enrolled patients where those missing a postbaseline assessment will be considered non-adherent. Also, a 'per protocol' analysis will be conducted after excluding intervention patients with no record of downloading any videos after randomisation. Finally, additional subgroup analyses will be performed after stratifying on baseline demographic and socioeconomic variables (age, sex, income, education attained, health literacy).

Secondary endpoints examining knowledge, beliefs about medications, quality of life, self-efficacy, satisfaction and days spent in hospital during the 1-year follow-up will be compared using t-tests for independent samples. In addition, predictors of optimal adherence (ie, the binary primary endpoint) independent of the video intervention will be examined using logistic regression analysis. The following independent variables collected at baseline will be examined in univariate analyses: demographics, health literacy, previous self-reported adherence, transplant knowledge, previous education, beliefs about medications and quality of life. Variables that are significant at $\mathrm{p}<0.20$ will be included in a multivariable model containing intervention group.

\section{ETHICS AND DISSEMINATION}

All patients provide informed consent prior to participating. The results of this study will be presented at conference proceedings and published in a peer-reviewed journal. The educational intervention aims to improve information retention and self-efficacy, leading to improved medication adherence after kidney transplantation, at low cost, and with little impact to existing healthcare personnel. If proven beneficial, delivery can be easily implemented into standard of care and readily provided to transplant patients across North America.

\section{Patient and public involvement}

The video series used in the study was developed with patients based on educational gaps identified by patients. This process involved a series of consultations with kidney transplant patients and their families to determine optimal content, after which 35 patients volunteered their time to be filmed for the videos. ${ }^{25}$ Finally an intensive review process was undertaken and the videos were modified accordingly. Two renal transplant recipients (PT and EW), were consultants throughout the development of this clinical trial protocol. They reviewed all procedures and documents and tested the burden of the intervention. When the study has been completed, the results will be disseminated on our study website.
Author affiliations

${ }^{1}$ College of Pharmacy and Nutrition, University of Saskatchewan, Saskatoon, Saskatchewan, Canada

${ }^{2}$ Saskatchewan Transplant Program, Saskatchewan Health Authority, Saskatoon, Saskatchewan, Canada

${ }^{3}$ Department of Surgery, University of Illinois Hospital and Health Sciences System, Chicago, Illinois, USA

${ }^{4}$ Southern Alberta Transplant Program, Alberta Health Services, Calgary, Alberta, Canada

${ }^{5}$ Multi-organ Transplant Program of Atlantic Canada, Halifax, Nova Scotia, Canada ${ }^{6}$ College of Medicine, University of Saskatchewan, Saskatoon, Saskatchewan, Canada

${ }^{7}$ College of Arts and Science, University of Saskatchewan, Saskatoon, SK, Canada

Acknowledgements The authors wish to thank Brianna Groot and Jessica McKutcheon at the Social Sciences Research Laboratory (SSRL) for their assistance with study design and execution. The authors would like to thank Paraag Trivedi and Errin Willenborg for reviewing the protocol from a patient perspective, and acknowledge all of the patients that participated in developing the video series used in this study.

Contributors NR, PT, JW, CD, RM, DB and AS participated in the design of the study and contributed to the revising the protocol manuscript. JL was responsible for the statistical design of the study. HM is the principal investigator of the study and is responsible for overseeing the project, including trial design and manuscript preparation. All authors approved the final manuscript.

Funding This work was supported by the American Society of Transplantation, Transplant and Immunology Research Network, grant internal ID 99399.

Competing interests HM, NR, RM and AS developed the video intervention used in this project.

Patient consent for publication Not required.

Ethics approval The protocol has been approved by the University of Saskatchewan Behavioural Ethics Board (Beh 18-63).

Provenance and peer review Not commissioned; externally peer reviewed.

Open access This is an open access article distributed in accordance with the Creative Commons Attribution Non Commercial (CC BY-NC 4.0) license, which permits others to distribute, remix, adapt, build upon this work non-commercially, and license their derivative works on different terms, provided the original work is properly cited, appropriate credit is given, any changes made indicated, and the use is non-commercial. See: http://creativecommons.org/licenses/by-nc/4.0/.

\section{REFERENCES}

1. Wolfe RA, Ashby VB, Milford EL, et al. Comparison of mortality in all patients on dialysis, patients on dialysis awaiting transplantation, and recipients of a first cadaveric transplant. $N$ Engl J Med 1999;341:1725-30.

2. Oniscu GC, Brown H, Forsythe JL. Impact of cadaveric renal transplantation on survival in patients listed for transplantation. J Am Soc Nephrol 2005;16:1859-65.

3. Abecassis M, Bartlett ST, Collins AJ, et al. Kidney transplantation as primary therapy for end-stage renal disease: a National Kidney Foundation/Kidney Disease Outcomes Quality Initiative (NKF/ KDOQITM) conference. Clin J Am Soc Nephrol 2008;3:471-80.

4. Halloran PF. Immunosuppressive drugs for kidney transplantation. $N$ Engl J Med Overseas Ed 2004;351:2715-29.

5. Dew MA, DiMartini AF, De Vito Dabbs A, et al. Rates and risk factors for nonadherence to the medical regimen after adult solid organ transplantation. Transplantation 2007;83:858-73.

6. Pinsky BW, Takemoto SK, Lentine KL, et al. Transplant outcomes and economic costs associated with patient noncompliance to immunosuppression. Am J Transplant 2009;9:2597-606.

7. UNOS Data. More than 30,000 transplants performed annually for first time in United States. 2016 https://optn.transplant.hrsa.gov/ news/more-than-30-000-transplants-performed-annually-for-firsttime-in-united-states/

8. Jamieson NJ, Hanson CS, Josephson MA, et al. Motivations, challenges, and attitudes to self-management in kidney transplant recipients: a systematic review of qualitative studies. Am J Kidney Dis 2016;67:461-78.

9. Urstad KH, Wahl AK, Andersen MH, et al. Renal recipients' educational experiences in the early post-operative phase-a qualitative study. Scand J Caring Sci 2012;26:635-42. 
10. Trivedi P, Rosaasen N, Mansell $\mathrm{H}$. The health-care provider's perspective of education before kidney transplantation. Prog Transplant 2016;26:322-7.

11. Urstad KH, Andersen MH, Øyen O, et al. Patients' level of knowledge measured five days after kidney transplantation. Clin Transplant 2011;25:646-52.

12. Gordon EJ, Wolf MS. Health literacy skills of kidney transplant recipients. Prog Transplant 2009;19:25-34.

13. Surman OS. Informed consent: what the patient heard. Transplant Proc 2013;45:3155-6.

14. Massey EK, Tielen M, Laging M, et al. The role of goal cognitions, illness perceptions and treatment beliefs in self-reported adherence after kidney transplantation: a cohort study. J Psychosom Res 2013;75:229-34.

15. Rodrigue JR, Paek MJ, Egbuna O, et al. Making house calls increases living donor inquiries and evaluations for blacks on the kidney transplant waiting list. Transplantation 2014;98:979-86.

16. Marlow NM, Kazley AS, Chavin KD, et al. A patient navigator and education program for increasing potential living donors: a comparative observational study. Clin Transplant 2016;30:619-27.

17. Boulware LE, Hill-Briggs F, Kraus ES, et al. Effectiveness of educational and social worker interventions to activate patients' discussion and pursuit of preemptive living donor kidney transplantation: a randomized controlled trial. Am J Kidney Dis 2013;61:476-86.

18. Arriola KR, Powell CL, Thompson NJ, et al. Living donor transplant education for African American patients with end-stage renal disease. Prog Transplant 2014;24:362-70.

19. Fahrenwald NL, Belitz C, Keckler A. Outcome evaluation of 'sharing the gift of life': an organ and tissue donation educational program for American Indians. Am J Transplant 2010;10:1453-9.

20. Nevins TE, Nickerson PW, Dew MA. Understanding medication nonadherence after kidney transplant. J Am Soc Nephrol 2017;28:2290-301.

21. Weng LC, Yang YC, Huang HL, et al. Factors that determine self-reported immunosuppressant adherence in kidney transplant recipients: a correlational study. J Adv Nurs 2017;73:228-39.

22. Silva AN, Moratelli L, Tavares PL, et al. Self-efficacy beliefs, locus of control, religiosity and non-adherence to immunosuppressive medications in kidney transplant patients. Nephrology 2016;21:938-43.

23. Chisholm-Burns MA, Spivey CA, Graff Zivin J, et al. Improving outcomes of renal transplant recipients with behavioral adherence contracts: a randomized controlled trial. Am J Transplant 2013:13:2364-73.

24. Skelton SL, Waterman AD, Davis LA, et al. Applying best practices to designing patient education for patients with end-stage renal disease pursuing kidney transplant. Prog Transplant 2015;25:77-90.

25. Rosaasen N, Mainra R, Kukha-Bryson A, et al. Development of a patient-centered video series to improve education before kidney transplantation. Patient Educ Couns 2018;101:1624-9.

26. Knowles M. Andragogy in action: applying modern principles of adult learning, jossey-bass. San Francisco, 1984

27. Pappas $\mathrm{C}$. The adult learning theory- andragog - of malcolm knowles. eLearning Industry. 2015. https://elearningindustry.com/ the-adult-learning-theory-andragogy-of-malcolm-knowles (Accessed 20th Oct 2015)

28. Gagliano ME. A literature review on the efficacy of video in patient education. J Med Educ 1988;63:785-92.

29. Kam J, Khadra S, Tran QH, et al. Portable video media versus standard verbal communication in surgical teaching: a prospective, multicenter, and randomized controlled crossover trial. J Surg Educ 2018;7204:30396-9.

30. Schotte C, Maes M, Beuten T, et al. A videotape as introduction for cognitive behavioral therapy with depressed inpatients. Psychol Rep 1993;72:440-2.
31. Gaskey NJ. Evaluation of the effect of a pre-operative anesthesia videotape. Aana J 1987;55:341-5

32. Minton PN. Video tape instruction: an effective way to learn. Rehabil Nurs 1983;8:15-17.

33. Weng FL, Brown DR, Peipert JD, et al. Protocol of a cluster randomized trial of an educational intervention to increase knowledge of living donor kidney transplant among potential transplant candidates. BMC Nephrol 2013;14:256.

34. Chew LD, Griffin JM, Partin MR, et al. Validation of screening questions for limited health literacy in a large VA outpatient population. J Gen Intern Med 2008;23:561-6.

35. Rosaasen N, Taylor J, Blackburn D, et al. Development and validation of the Kidney Transplant Understanding Tool (K-TUT). Transplant Direct 2017;3:e132.

36. Schwarzer R, Jerusalem M. Generalized Self-Efficacy scale. In: Weinman I, Wright S, Johnston M, Measures in health psychology: a user's portfolio. causal and control beliefs. Windsor: NEFR Nelson, 1995.

37. Horne R, Weinman J, Hankins M. The beliefs about medicines questionnaire: the development and evaluation of a new method for assessing the cognitive representation of medication. Psychol Health 1999;14:1-24.

38. Ware J, Kosinski M, Keller SD. A 12-Item Short-Form Health Survey: construction of scales and preliminary tests of reliability and validity. Med Care 1996;34:220-33.

39. Chisholm-Burns MA, Spivey CA, Sredzinski E, et al. Intervention toolbox to promote immunosuppressant therapy adherence in adult renal transplant recipients. J Am Pharm Assoc 2012;52:816-22.

40. Jones J, Rosaasen N, Taylor J, et al. Health literacy, knowledge, and patient satisfaction before kidney transplantation. Transplant Proc 2016:48:2608-14.

41. Dobbels F, Berben L, De Geest S, et al. The psychometric properties and practicability of self-report instruments to identify medication nonadherence in adult transplant patients: a systematic review. Transplantation 2010;90:205-19.

42. Hsiau M, Fernandez HE, Gjertson D, et al. Monitoring nonadherence and acute rejection with variation in blood immunosuppressant levels in pediatric renal transplantation. Transplantation 2011;92:918-22.

43. Scheel J, Reber S, Stoessel L, et al. Patient-reported non-adherence and immunosuppressant trough levels are associated with rejection after renal transplantation. BMC Nephrol 2017;18:107.

44. Urstad $\mathrm{KH}$, Øyen $\mathrm{O}$, Andersen $\mathrm{MH}$, et al. The effect of an educational intervention for renal recipients: a randomized controlled trial. Clin Transplant 2012;26:E246-53.

45. Zawadzka B, Zawadzka S, Sułowicz W, et al. Cooperation in Treatment as an Indicator of Adaptation of Kidney Transplant Patients to Chronic Therapy. Transplant Proc 2016;48:1598-603.

46. De las Cuevas C, Rivero-Santana A, Perestelo-Perez L, et al. Adaptation and validation study of the Beliefs about Medicines Questionnaire in psychiatric outpatients in a community mental health setting. Hum Psychopharmacol 2011;26:140-6.

47. Prasad GV, Nash MM, Keough-Ryan T, et al. A quality of life comparison in cyclosporine- and tacrolimus-treated renal transplant recipients across Canada. J Nephrol 2010;23:274-81.

48. Hathaway D, Winsett R, Prendergast $M$, et al. The first report from the patient outcomes registry for transplant effects on life (PORTEL): differences in side-effects and quality of life by organ type, time since transplant and immunosuppressive regimens. Clin Transplant 2003;17:183-94.

49. Toledo AH, Hendrix L, Buchholz V, et al. Improvement of gastrointestinal symptoms after conversion from mycophenolate mofetil to enteric-coated mycophenolate sodium in liver transplant patients. Clin Transplant 2012;26:156-63.

50. Taber DJ, Fleming JN, Fominaya CE, et al. The impact of health care appointment non-adherence on graft outcomes in kidney transplantation. Am J Nephrol 2017;45:91-8. 